\title{
GENERALISED INVERSE SEMIGROUPS WITH THE SMALLEST ELEMENT
}

\author{
Jolanta Lipińska \\ Institute of Mathematics, Czestochowa University of Technology \\ Częstochowa, Poland \\ jolanta.lipinska@im.pcz.pl
}

\begin{abstract}
In this paper we consider properties of generalised inverse semigroups with the smallest element. We show that if a generalised inverse semigroup has the smallest element, and it consists of at least two elements, we can leave the smallest element and we obtain a new generalised inverse semigroup. We show also examples of generalised inverse semigroups which have not the smallest element.
\end{abstract}

\section{Introduction}

The notion of a pseudogroup was formed progressively together with the development of differential geometry. The first mathematicians who realized that a classic notion of a group of transformations was not sufficient for purposes of differential geometry were O. Veblen and J.H.C. Whitehead in 1932. Their definition was improved by J.A. Schouten and J. Haantjes in 1937, S. Gołąb in 1939 and C. Ehresmann in 1947. Gołąb's and Ehresmann's definitions are good enough to be used at present. It was shown in [1] that axioms of Ehresmann's definition can be formulated in the equivalent way. We used this definition in [2] to show that a group of transformations can be treated as a pseudogroup.

\section{Main result}

Let us recall the following version of Ehresmann's definition which is used in differential geometry and can be found in [3].

Definition 1. A pseudogroup of transformations on a topological space $S$ is a set $\Gamma$ of transformations satisfying the following axioms:

$1^{\circ}$ Each $f \in \Gamma$ is a homeomorphism of an open set of $S$ onto another open set of $S$;

$2^{\circ}$ If $f \in \Gamma$, then the restriction of $f$ to an arbitrary open subset of the domain of $f$ is in $\Gamma$; 
$3^{\circ}$ Let $U=\bigcup_{i} U_{i}$ where each $U_{i}$ is an open set of $S$. A homeomorphism $f$ of $U$ onto an open set of $S$ belongs to $\Gamma$ if the restriction of $f$ to $U_{i}$ is in $\Gamma$ for every $i$;

$4^{\circ}$ For every open set $U$ of $S$, the identity transformation of $U$ is in $\Gamma$;

$5^{\circ}$ If $f \in \Gamma$, then $f^{-1} \in \Gamma$;

$6^{\circ}$ If $f \in \Gamma$ is a homeomorphism of $U$ onto $V$ and $g \in \Gamma$ is a homeomorphism of $Y$ onto $Z$ and if $V \cap Y$ is non-empty, then the homeomorphism $g \circ f$ of $f^{-1}(V \cap Y)$ onto $g(V \cap Y)$ is in $\Gamma$.

We will also use the following definition introduced in [1].

Definition 2. A non-empty set $\Gamma$ of functions, for which domains $D_{f}$ are arbitrary non-empty sets, will be called a pseudogroup of functions if it satisfies the following conditions:

$1^{\circ} f\left(D_{f}\right) \cap D_{g} \neq \varnothing \Rightarrow g \circ f \in \Gamma$ for $f, g \in \Gamma$,

$2^{\circ} f^{-1} \in \Gamma$ for $f \in \Gamma$,

$3^{\circ} \cup \Gamma^{\prime} \in \Gamma$ for $\Gamma^{\prime} \in\langle\Gamma\rangle$

where

$$
\langle\Gamma\rangle=\left\{\varnothing \neq \Gamma^{\prime} \subset \Gamma: \cup \Gamma^{\prime} \text { is a function and } \bigcup\left(\Gamma^{\prime}\right)^{-1} \text { is a function }\right\}
$$

and

$$
\left(\Gamma^{\prime}\right)^{-1}=\left\{f^{-1}: f \in \Gamma^{\prime}\right\}
$$

and $f^{-1}$ denotes an inverse relation.

It was shown in [1] that if $\Gamma$ is a pseudogroup, then $\left(\bigcup_{f \in \Gamma} D_{f},\left\{D_{f}: f \in \Gamma\right\} \cup\{\varnothing\}\right)$ is a topological space and $\Gamma$ is an Ehresmann pseudogroup of transformations on this topological space. On the other hand, if $\Gamma$ is an Ehresmann pseudogroup of transformations on a topological space $S$, then $\Gamma$ is a pseudogroup of functions.

We will need the following definition which was introduced in [4].

Definition 3. A generalized inverse semigroup is a partial groupoid $(B, \bullet)$ satisfying the following axioms:

$1^{\circ} a \bullet(b \bullet c)=(a \bullet b) \bullet c$

holds when one of the sides is defined; 
$2^{\circ}$ For every $a \in B$ there exists exactly one $b \in B$ such that

$$
a \bullet(b \bullet a)=a \quad \text { and } \quad b \bullet(a \bullet b)=b
$$

We will also need for elements of a generalized inverse semigroup $(B, \bullet)$ the following definitions and denotations which were introduced in [4]. We will write $a b$ instead of $a \bullet b$. For every $a \in B$ the only one $b \in B$ from $2^{\circ}$ of Definition 3 will be denoted by $a^{\prime}$ and called a generalised inverse element of $a, a^{\prime} a$ will be called a right identity of $a$ and $a a^{\prime}$ a left identity of $a$. It is obvious that $a$ will be then a generalised inverse element of $a^{\prime}, a^{\prime} a$ will be a left identity of $a^{\prime}$ and $a a^{\prime}$ a right identity of $a^{\prime}$. If $a$ is a right and left identity for all elements of $B$ we say that $a$ is an identity. We will say that $a \in B$ is an idempotent element when $a a=a$. It was shown in [4] that $a=a^{\prime}$ for an idempotent element $a$ so it means that its generalised inverse element, right and left identity, are all equal to $a$. It was also shown that the following relation

$$
a \leq b \Leftrightarrow b a^{\prime} a=a
$$

is a partial order in a generalized inverse semigroup. To prove it we used a lemma saying that if $a, b$ are idempotent elements the operation $a b$ is commutative.

It was proved in [5] that we can obtain an inverse semigroup from every generalised inverse semigroup $(B, \bullet)$ joining an element $O \notin B$. Then $(B \cup\{O\}, *)$ is a semigroup where the operation $*$ is defined in the following way:

$$
\begin{gathered}
a * b=a b \text { when the operation } a b \text { is defined } \\
\qquad O \text { in the other case }
\end{gathered}
$$

We will also need the theorem which was proved in [6] and says that if $\Gamma$ is a pseudogroup of transformations on a topological space $S$, then $\Gamma$ is a generalised inverse semigroup with identity. Of course we can replace a pseudogroup of transformations by a pseudogroup of functions and the theorem will be true.

Now we will formulate the problem. We can obtain an inverse semigroup from every generalised inverse semigroup $(B, \bullet)$ joining an element $O \notin B$. The problem is when it is possible to leave an element which belongs to a generalised inverse semigroup to obtain a new generalised inverse semigroup. We can formulate the following theorem.

Theorem 1. If a generalised inverse semigroup has the smallest element and it consists of at least two elements we can leave the smallest element and we obtain a new generalised inverse semigroup.

Proof. It is easy to show that the smallest element of a generalised inverse semigroup is an idempotent element. Let us denote the smallest element by $a$. We 
know that every $b$ which belongs to the generalised inverse semigroup $(B, \bullet)$ satisfies the equation

$$
b a^{\prime} a=a
$$

as the relation of partial order is satisfied. We can put $b=a^{\prime} a$. We obtain

$$
a^{\prime} a a^{\prime} a=a
$$

But $a^{\prime} a$ is an idempotent element as it is a right identity for $a$. Hence we have

$$
a^{\prime} a=a
$$

As $a$ is an idempotent element, its right identity, left identity and inverse element are equal to it. So we can leave it. We obtain a new generalised inverse semigroup. Of course, an inverse semigroup is also a generalised inverse semigroup, so we can use the theorem for it.

The next problem appears if all generalised inverse semigroups have the smallest element. We show that it is not true.

Example 1. Let us consider the set which consists of two functions: identity defined on $(0, \div \infty)$ and $\frac{1}{x}$ defined also on $(0, \div \infty)$. It is an inverse semigroup and there is not the smallest element.

Proof. As it was shown in [7] it is an inverse semigroup. We can even add that it is a group. We can see that the relation of partial order does not hold. That is required.

Of course when a group consists of at least two elements there is not the smallest element so we will provide the next example.

Example 2. Let us consider the set which consists of identities defined on intervals $(-1-r, 1+r)$ where $r$ belongs to $[0,1]$. It is an inverse semigroup. There is the smallest element but we can leave the smallest element and we get a new inverse semigroup where there is not the smallest element.

Proof. We can use Theorem 1.

\section{Conclusions}

In [7] we considered properties of a set of idempotent elements of generalised inverse semigroups. Now we considered properties of generalised inverse semigroups with the smallest element. We proved that if a generalised inverse semigroup consists of at least two elements, and has the smallest element, we can leave 
the smallest element and we get a new generalised inverse semigroup. We gave two examples where the smallest element does not exist.

\section{References}

[1] Lipińska J., Diffeomorphisms of quasi-algebraic spaces, Demonstratio Mat. 1986, 19, 139-151.

[2] Lipińska J., Groups of transformations as pseudogroups of functions, Scientific Research of the Institute of Mathematics and Computer Science 2006, 1(5), 69-70.

[3] Kobayashi S., Nomizu K., Foundations of Differential Geometry I, New York, London 1963.

[4] Lipińska J., Uogólniona półgrupa inwersyjna i jej własności, Zeszyty Naukowe Pol. Śl. Mat.-Fiz. 1981, 39, 287-295 (in Polish).

[5] Lipińska J., Stosunek uogólnionej „półgrupy” inwersyjnej do innych systemów algebraicznych z jedną dwuargumentową częściową operacją, Zeszyty Naukowe Pol. Śl. Mat.-Fiz. 1981, 39, 305-310 (in Polish).

[6] Lipińska J., Przykłady uogólnionych „półgrup” inwersyjnych, Zeszyty Naukowe Pol. Śl. Mat.-Fiz. 1981, 39, 297-303 (in Polish).

[7] Lipińska J., Properties of a set of idempotent elements in generalised inverse semigroups, Scientific Research of the Institute of Mathematics and Computer Science 2012, 3(11), 93-97. 